10

\title{
Динамика решеток и барическое поведение фононов в модельных сегнетоэластиках $\mathrm{Hg}_{2} \mathrm{Br}_{2}$
}

\author{
(C) Е.М. Рогинский, ${ }^{1}$ А.С. Крылов, ${ }^{2}$ Ю.Ф. Марков ${ }^{1}$ \\ ${ }^{1}$ Физико-технический институт им. А.Ф. Иофрфе РАН, \\ Санкт-Петербург, Россия \\ ${ }^{2}$ Институт физики им. Л.В. Киренского СО РАН, \\ Красноярск, Россия \\ E-mail: e.roginskii@mail.ioffe.ru
}

(Поступила в Редакцию 18 июля 2018 г.)

В широком интервале высоких гидростатических давлений изучены спектры комбинационного рассеяния модельных несобственных сегнетоэластиков - кристаллов $\mathrm{Hg}_{2} \mathrm{Br}_{2}$. Получены барические зависимости частот фононов, среди которых наибольший интерес вызывает обнаружение и аномальное поведение мягкой моды, генетически связанной с наиболее медленной акустической ветвью $T A_{1}$ на границе зоны Бриллюэна ( $X$-точка) тетрагональной фазы. В спектрах сегнетоэластической фазы обнаружено также возгорание второй акустики $\left(T A_{2}\right)$ из этой же точки и изучено ее барическое поведение. При достаточно больших давлениях наблюдалось и было объяснено расщепление дважды вырожденных фононов симметрии $E_{g}$. Из барических зависимостей частот фононов получены и обсуждены параметры констант Грюнайзена.

Работа выполнена при частичной финансовой поддержке программ Президиума РАН: „Актуальные проблемы физики низких температур“ № 1.4 и „Физика конденсированных сред и материалы нового поколения““ № 1.7 .

DOI: 10.21883/FTT.2019.02.47133.208

\section{1. Введение}

Кристаллы галогенидов одновалентной ртути $\mathrm{Hg}_{2} \mathrm{Hal}_{2}$ $(\mathrm{Hal}=\mathrm{F}, \mathrm{Cl}, \mathrm{Br}, \mathrm{I})$ при комнатной температуре имеют очень простую структуру, состоящую из параллельных оптической оси $C_{4}(Z)$ цепочек линейных молекул $\mathrm{Hal}-\mathrm{Hg}-\mathrm{Hg}-\mathrm{Hal}$, слабо связанных друг с другом и образующих объемно-центрированную тетрагональную решетку $D_{4 h}^{17}$ с двумя молекулами в элементарной ячейке (рис. 1) [1]. Цепочечное строение этих кристаллов приводит к очень сильной анизотропии их физических свойств, в том числе упругих, оптических и др., Эти кристаллы демонстрируют уникальные физические свойства например, они имеют рекордно низкие среди твердых тел скорости поперечного $(T A)$ звука $V_{[110]}^{[11 \overline{0}]}=282 \mathrm{~m} / \mathrm{s}$ $\left(\mathrm{Hg}_{2} \mathrm{Br}_{2}\right)$ и $253 \mathrm{~m} / \mathrm{s} \quad\left(\mathrm{Hg}_{2} \mathrm{I}_{2}\right)$, рекордно высокие значения двулучепреломления $\Delta n=+0.85\left(\mathrm{Hg}_{2} \mathrm{Br}_{2}\right)$, $+1.48\left(\mathrm{Hg}_{2} \mathrm{I}_{2}\right)$ и акусто-оптическое взаимодействие для $T A$-волны $M_{2}=1804 \cdot 10^{-18}$ units CGS $\left(\mathrm{Hg}_{2} \mathrm{Br}_{2}\right)$ и $4284 \cdot 10^{-18}$ units CGS $\left(\mathrm{Hg}_{2} \mathrm{I}_{2}\right)$ [2].

Вышеуказанные уникальные свойства позволяют успешно использовать эти кристаллы в технике в качестве основных элементов поляризаторов, акустических линий задержки, акустооптических фильтров, модуляторов, дефлекторов и др.

Значительный интерес к этому семейству изоморфных кристаллов связан также с тем, что они являются модельными кристаллическими системами при исследовании общих проблем структурных фазовых переходов (ФП). При охлаждении до $T_{c}=186 \mathrm{~K}\left(\mathrm{Hg}_{2} \mathrm{Cl}_{2}\right)$ и
$T_{c}=144 \mathrm{~K}\left(\mathrm{Hg}_{2} \mathrm{Br}_{2}\right)$ эти кристаллы испытывают несобственные сегнетоэластические $\Phi П D_{4 h}^{17} \rightarrow D_{2 h}^{17}$ из тетрагональной фазы в ромбическую. ФП индуцированы конденсацией наиболее „медленной“, наиболее низкочастотной $T A$-ветви в $X$-точке границы зоны Бриллюэна (ЗБ) тетрагональной парафазы и сопровождаются при $T \leq T_{c}$ удвоением элементарной ячейки, $X \rightarrow \Gamma$ „перебросом“ в ЗБ, возникновением спонтанной деформации и сегнетоэластических доменов [3-5].

Однако в изоморфных кристаллах $\mathrm{Hg}_{2} \mathrm{I}_{2}$ ФП при атмосферном давлении не происходит даже при охлаждении до очень низких температур, вплоть до $1.5 \mathrm{~K}$, но в спектрах комбинационного рассеяния света наблюдалось смягчение одного из малоинтенсивных низкочастотных колебаний [6]. По аналогии с похожими эффектами в изоморфных кристаллах $\mathrm{Hg}_{2} \mathrm{Cl}_{2}$ и $\mathrm{Hg}_{2} \mathrm{Br}_{2}$ предполагалось, что это колебание является обертоном мягкой $T A$-ветви с границы ЗБ ( $X$-точка). При атмосферном давлении кристаллы $\mathrm{Hg}_{2} \mathrm{I}_{2}$ являются виртуальными сегнетоэластиками $\left(T_{C} \approx-20 \mathrm{~K}\right)$. ФП в этих кристаллах удалось реализовать лишь при высоком гидростатическом давлении $9 \operatorname{kbar}(T=300 \mathrm{~K})$ [7]. Значительное внимание было уделено изучению барического поведения мягких мод, как в парафазе $\left(P<P_{c}\right)$, так и в сегнетоэластической фазе $\left(P>P_{c}\right)[8,9]$.

Недавно, теоретически и экспериментально, нами изучался изоморфный и также модельный сегнетоэластик $\mathrm{Hg}_{2} \mathrm{Cl}_{2}$ [10]. В этой работе было изучено барическое поведение фононов, в том числе мягких, а также 
обнаружен принципиальный эффект, а именно новый структурный фазовый переход.

В настоящей работе продолжены эти барические исследования, но уже на других, также модельных и изоморфных кристаллах $\mathrm{Hg}_{2} \mathrm{Br}_{2}$.

\section{2. Методика эксперимента}

Эксперименты в условиях высокого гидростатического давления (до $13 \mathrm{GPa}$ ) проводились на установке с алмазными наковальнями при температуре $295 \mathrm{~K}$. Диаметр камеры с образцом $0.25 \mathrm{~mm}$, высота $0.1 \mathrm{~mm}$. В качестве передающей давление среды использовались различные масла (при давлениях до 4-5 GPa), а при более высоких давлениях - тщательно обезвоженная смесь этилового и метилового спиртов. Давление в „алмазной“ камере определялось по сдвигу полосы люминесценции рубина, микрокристалл которого помещался вблизи изучаемого образца [11]. Погрешность измеряемого давления не превышала $0.05 \mathrm{GPa}$.

При изучении спектров комбинационного рассеяния (КР), в качестве источника возбуждения, было использовано поляризованное излучение $514.5 \mathrm{~nm}$ Ar-лазера (Spectra-Physics Stabilite 2017) мощностью $100 \mathrm{~mW}$ (20 mW на образце). Спектры в геометрии $180^{\circ}$ регистрировались с помощью спектрометра Horiba Jobin Yvon T64000 в частотном диапазоне $20-400 \mathrm{~cm}^{-1}$.

Предварительно изучаемые кристаллы $\mathrm{Hg}_{2} \mathrm{Br}_{2}$ раскалывались по плоскостям спайности $\{110\}$ и $\{1 \overline{1} 0\}$, из множества сколотых образцов для измерений подбирались монокристаллы необходимого размера. Изучаемые образцы размером не более $\sim 0.2 \times 0.2 \times 0.2 \mathrm{~mm}$ помещались в камеру с алмазными наковальнями, позволяющую проводить оптические исследования, в том числе поляризационные при давлениях от 0.2 до $13 \mathrm{GPa}$.

\section{3. Экспериментальные результаты и их обсуждение}

Примитивная ячейка кристаллов $\mathrm{Hg}_{2} \mathrm{Br}_{2}$ тетрагональной фазы (структура с группой пространственной симметрии $\left.D_{4 h}^{17}\right)$ состоит всего из одной линейной молекулы (4 атома), поэтому, согласно теории групп, разложение колебательного спектра по неприводимым представлениям (НП) имеет вид

$$
\Gamma=2 A_{1 g}+2 A_{2 u}+2 E_{u}+2 E_{g} .
$$

При этом в спектрах КР активными будут лишь 2 колебания симметрии $A_{1 g}$ и 2 колебания симметрии $E_{g}$. Собственные векторы (нормальные координаты) этих колебаний приведены на рис. 1. Низкочастотные колебания, обозначенные на рис. 1 как $v_{1}$ и $v_{2}$, обладают симметрией $E_{g}$ и поэтому будут активны в спектрах КР в поляризациях $X Z(Y Z)$, полносимметричные колебания симметрии $A_{1 g}\left(v_{3}\right.$ и $\left.v_{4}\right)$ разрешены в спектрах КР в поляризациях $X X, Y Y$ и $Z Z$.

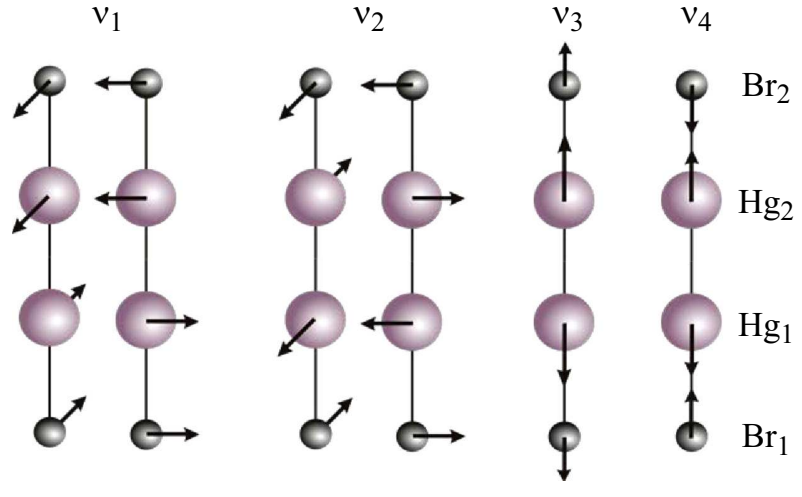

Рис. 1. Векторы нормальных колебаний тетрагональной фазы $D_{4 h}^{17}\left(\mathrm{Hg}_{2} \mathrm{Br}_{2}\right)$.

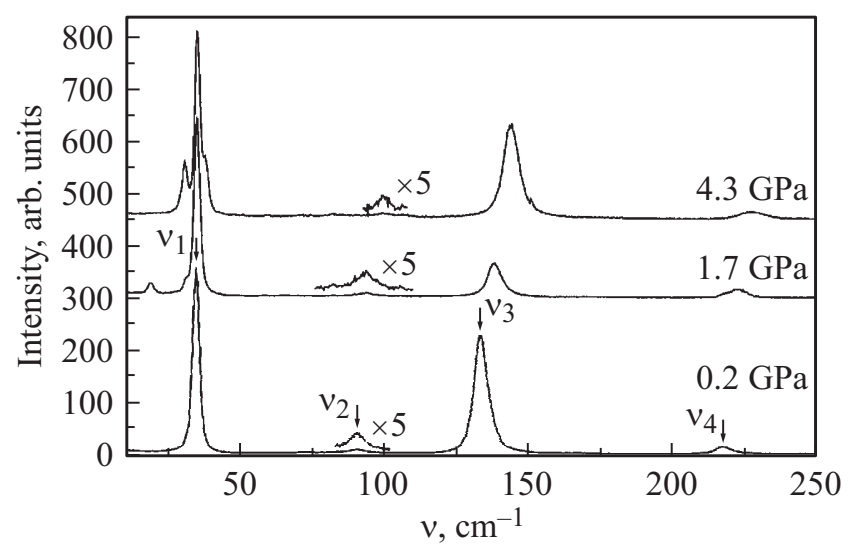

Рис. 2. Спектры комбинационного рассеяния (КР) кристаллов $\mathrm{Hg}_{2} \mathrm{Br}_{2}$ при низком $(0.2 \mathrm{GPa})$ и высоких гидростатических $(1.7 \mathrm{GPa}$ и $4.3 \mathrm{GPa})$ давлениях.

Следует заметить, что первое дважды вырожденное колебание симметрии $E_{g}-$ это либрация, качание линейной молекулы как целого относительно горизонтальной оси $X$ (или $Y$ ), обозначенное как $v_{1}$; второе колебание симметрии $E_{g}$ - деформационное „зигзагообразное $\left(v_{2}\right)$; полносимметричные валентные колебания $A_{1 g}$ соответствуют, главным образом, смещениям $\mathrm{Hg}-\mathrm{Hg}\left(v_{3}\right)$ и $\mathrm{Br}-\mathrm{Hg}\left(v_{4}\right)$ (см. рис. 1$)$.

На рис. 2 приведены спектры кристаллов $\mathrm{Hg}_{2} \mathrm{Br}_{2}$ при различных гидростатических давлениях $(0.2,1.7$ и $4.3 \mathrm{GPa})$. Во всех приведенных на рис. 2 спектрах наблюдается две линии, соответствующие колебаниям симметрии $E_{g}$ и две линии симметрии $A_{1 g}$, что коррелирует с результатами теоретико-группового анализа. Однако в спектрах образцов, испытывающих давление выше $0.2 \mathrm{GPa}$, наблюдаются особенности, связанные со структурным фазовым переходом $D_{4 h}^{17} \rightarrow D_{2 h}^{17}$, в частности наблюдаются значительные и соизмеримые сдвиги частот $v_{2}, v_{3}$ и $v_{4}$, в спектрах появляются новые линии вследствие удвоения элементарной ячейки, а в области либрационного колебания $v_{1}$ убедительно проявляются как небольшие сдвиги частот фононов, так и их аномаль- 
ное поведение, в том числе расщепление вырожденных колебаний $v_{1}\left(E_{g}\right)$ (см. рис. 2,3$)$.

Следует напомнить, что фазовый переход $D_{4 h}^{17} \rightarrow D_{2 h}^{17}$ индуцирован конденсацией поперечного $T A_{1}$ фонона на границе $X$-точка ЗБ, неприводимое представление которого $X_{3}^{-}$. В таблице согласно результатам теоретикогруппового анализа, выполненного с применением программ сервера Bilbao Crystallographic Server (BCS) [12], построено соответствие неприводимых представлений фононов тетрагональной $\left(D_{4 h}^{17}\right)$ и ромбической $\left(D_{2 h}^{17}\right)$ фаз. Из этой таблицы, в частности, видно, что в результате фазового перехода снимается вырождение поперечных акустических и оптических фононов $v_{1}, v_{5}$. Согласно таблице, НП $E_{g}$ тетрагональной фазы „разбивается“ на два НП $B_{1 g}+B_{2 g}$, а НП $E_{u}$ тетрагональной фазы „разбивается" на два НП $B_{1 u}+B_{2 u}$. Кроме того, в результате $X \rightarrow \Gamma$ „переброса“ в центре ЗБ ромбической фазы появляются новые фононы, НП которых $A_{u}, B_{2 u}, B_{3 u}$, $A_{g}, B_{1 g}$ и $B_{3 g}$, что приводит к появлению новых линий в спектрах КР. Ярким примером такого обогащения спектра может служить фонон симметрии $A_{g}$ ромбической фазы, который генетически связан с поперечным акустическим колебанием $T A_{2}$ тетрагональной фазы (мягкая мода) и фонон симметрии $B_{3 g}$, генетически связанный со вторым поперечным акустическим фононом на границе ЗБ тетрагональной фазы.

Для иллюстрации эффектов фазового перехода в этих кристаллах на рис. 3 построены спектры КР при различных гидростатических давлениях в области низких частот $\left(0-50 \mathrm{~cm}^{-1}\right)$. Наиболее интересным при изучении динамики решетки кристаллов $\mathrm{Hg}_{2} \mathrm{Br}_{2}$ при высоких гидростатических давлениях оказалось значительное ужесточение при увеличении давления наиболее низкочастотного $A_{g}$ фонона, обозначенного на рис. 3 $v_{s m}\left(T A_{1}\right)$, генезис которого обсуждался выше.

Также стоит отметить появление в спектре КР-фонона симметрии $B_{1 g}$ на низкочастотном крыле линии, связанной с либрационным колебанием $v_{1}$ (см. рис. 2,3). Появление этого максимума также обсуждалось выше, а

\begin{tabular}{|c|c|c|c|}
\hline Мода & $D_{4 h}^{17}, \Gamma$ & $D_{4 h}^{17}, X$ & $D_{2 h}^{17}, \Gamma$ \\
\hline \multirow{3}{*}{$v_{1}$} & $E_{g}$ & & $B_{1 g}+B_{2 g}$ \\
\hline & & $X_{3}^{+}$ & $A_{u}$ \\
\hline & & $X_{4}^{+}$ & $B_{3 u}$ \\
\hline \multirow{2}{*}{$v_{3,4}$} & $A_{1 g}$ & & $A_{g}$ \\
\hline & & $X_{1}^{+}$ & $B_{2 u}$ \\
\hline \multirow{3}{*}{$v_{5}+T A_{1}+L A$} & $E_{u}$ & & $B_{1 u}+B_{2 u}$ \\
\hline & & $X_{3}^{-}$ & $A_{g}$ \\
\hline & & $X_{4}^{-}$ & $B_{3 g}$ \\
\hline \multirow{2}{*}{$v_{6}+T A_{2}$} & $A_{2 u}$ & & $B_{3 u}$ \\
\hline & & $X_{2}^{-}$ & $B_{1 g}$ \\
\hline
\end{tabular}

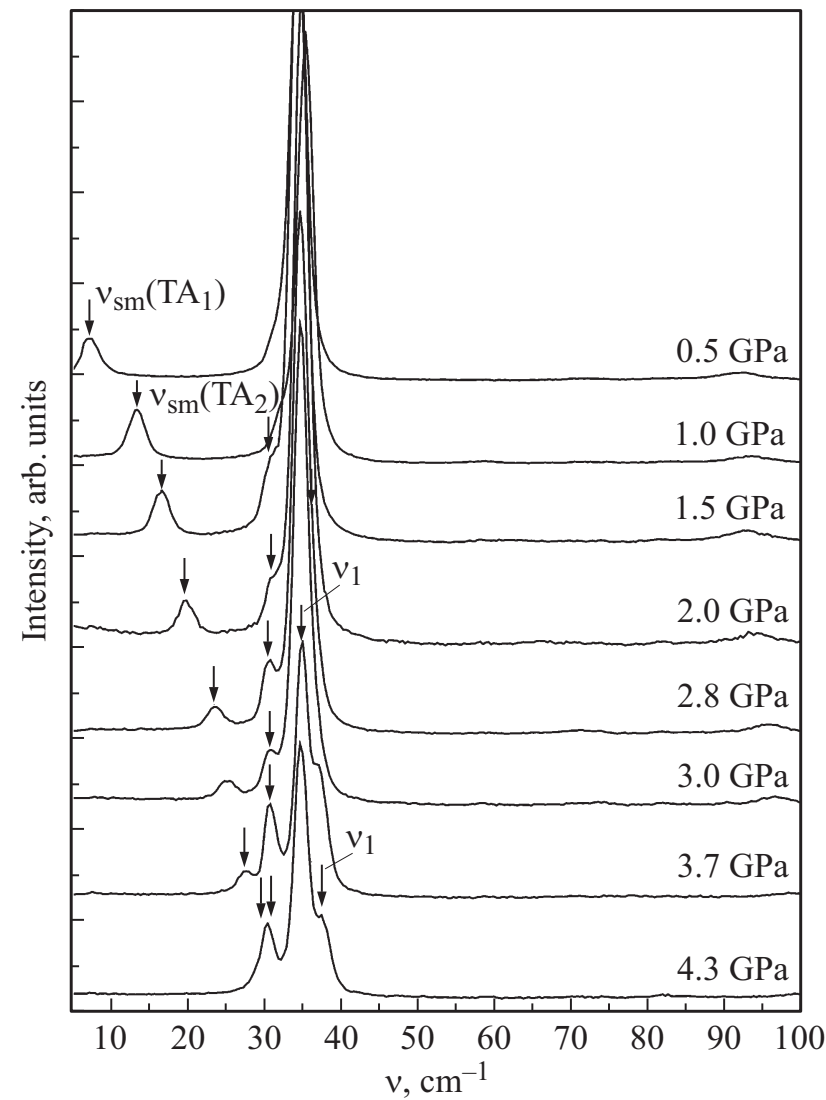

Рис. 3. Низкочастотные спектры КР кристаллов $\mathrm{Hg}_{2} \mathrm{Br}_{2}$ при различных гидростатических давлениях.

зависимость частоты этого фонона от давления можно описать как незначительное смягчение.

В спектрах КР можно было бы обнаружить максимумы, связанные с двухфононным взаимодействием, и ИК-активные фононы, как, например, в случае кристаллов $\mathrm{Hg}_{2} \mathrm{I}_{2}$ [7,8]. Но это задача достаточно сложная, так как эксперименты в алмазной камере с маленькими кристалликами и малой апертурой камеры в разы понижают полезный сигнал. Кроме этого, нельзя не учитывать влияние полидоменности изучаемых образцов, а также значительный сдвиг края поглощения в низкочастотную область спектра, понижающий прозрачность исследуемых образцов при возрастании давления в камере.

При давлениях выше $3 \mathrm{GPa}$ в спектрах наблюдается расщепление дважды вырожденного колебания $v_{1}$ на две компоненты (линии, соответствующие фононам симметрии $B_{1 g}$ и $B_{2 g}$, см. таблицу), связанного с структурным фазовым переходом и потерей оси четвертого порядка.

На рис. 4 приведены зависимости частот фундаментальных колебаний в кристаллах $\mathrm{Hg}_{2} \mathrm{Br}_{2}$ от приложенных гидростатических давлений, которые удается c хорошей точностью описать линейной аппроксимацией, что позволяет определить динамику изменения частоты для каждого фонона активного в спектрах КР. 


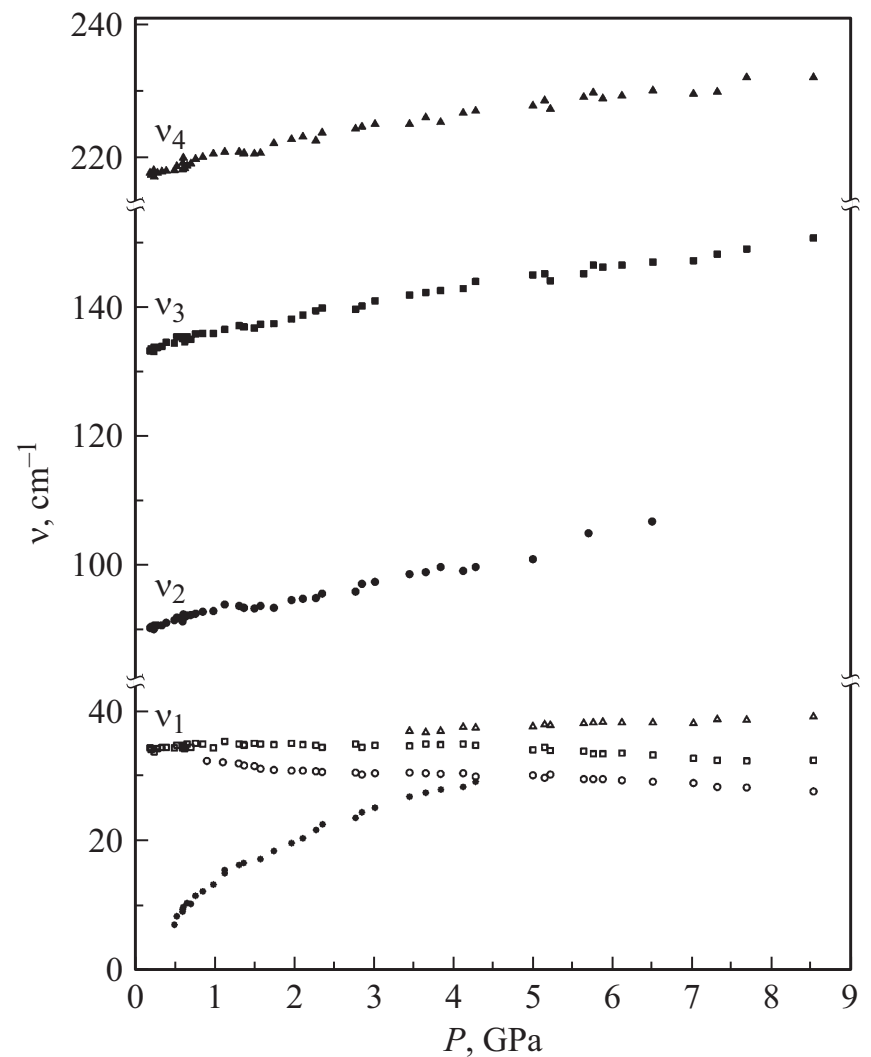

Рис. 4. Барические зависимости частот фононов в кристаллах $\mathrm{Hg}_{2} \mathrm{Br}_{2}$. Звездочки соответствуют частоте моды $v_{\mathrm{sm}}\left(T A_{1}\right)$, полые кружки - частота моды $v\left(T A_{2}\right)$, полые квадраты и треугольники - либрационное колебание $v_{1}$, сплошные кружки - деформационное колебание $v_{2}$, сплошные квадраты и треугольники - валентные колебания $v_{3}$ и $v_{4}$ соответственно.

Так, скорость изменения колебания $v_{\mathrm{sm}}\left(T A_{1}\right)$ составила $-0.38 \mathrm{~cm}^{-1} / \mathrm{GPa}$, для либрационного колебания $v_{i}$ и $v_{1}$ скорость равна -0.2 и $0.4 \mathrm{~cm}^{-1} / \mathrm{GPa}$ соответственно, для деформационного колебания $v_{2}$ она составила $2.0 \mathrm{~cm}^{-1} / \mathrm{GPa}$, и наконец для валентных колебаний $v_{3}$ и $v_{4} 2.05$ и $1.87 \mathrm{~cm}^{-1} / \mathrm{GPa}$ соответственно.

Следует заметить, что частоты фонона $v_{1}$ почти не зависят от давления, то есть константы Грюнайзена, характеризующие его барическое поведение, очень малы и даже отрицательны. Похожие эффекты также имели место в случае кристаллов $\mathrm{Hg}_{2} \mathrm{I}_{2}$ [9]. Подобное аномальное поведение можно связать с характером данного колебания. Как было показано в работе [13], кристаллы $\mathrm{Hg}_{2} \mathrm{Hal}_{2}$ - квазимолекулярные соединения, поэтому помимо кулоновского взаимодействия в данных кристаллах участвует дальнодействующее взаимодействие (например, силы Ван-дер-Ваальса). Наибольшее кулоновское взаимодействие происходит между молекулами, находящимися в узлах и объеме элементарной ячейки, поскольку находятся ближе всего друг к другу. Более того, кулоновское взаимодействие происходит главным образом между ближайшими половинами молекул в узлах и объеме $(\mathrm{Hg}-\mathrm{Br})$. В случае либрационного ко- лебания эти половины ближайших молекул сближаются максимально в фазе и удаляются в противофазе, при этом возникает конкурирующее взаимное притяжение катион-анион и отталкивание катион-катион (анионанион) соседних половин молекул, в результате чего это взаимодействие компенсируется и сближение молекул, как результат приложенного давления, не оказывает существенного влияния на изменение силовых констант, и даже ведет к их уменьшению, а следовательно к уменьшению частоты этого колебания.

Совсем другую динамику проявляют колебания $v_{2}$, $v_{3}, v_{4}$, демонстрируя сильные сдвиги частот при увеличении гидростатического давления (см. рис. 2) и, соответственно, большие и положительные значения констант Грюнайзена. Эти большие сдвиги частот можно попытаться связать с сильным влиянием приложенного гидростатического давления на ионно-ковалентные связи, существующие внутри молекул и приводящие к аномальному росту внутримолекулярных силовых констант $(v \sim \sqrt{k / \mu})$.

На рис. 5 приведена барическая зависимость частоты фонона $v_{\text {textcm }}$, которая может быть описана формулой $v_{\mathrm{sm}} \sim\left[\left(P-P_{c}\right) / P_{c}\right]^{\beta}$, где $\left[\left(P-P_{c}\right) / P_{c}\right]=p-$ приведенное давление, а значение давления фазового перехода при комнатной температуре $P_{c}=0.3 \mathrm{GPa}$. Чтобы определить достаточно строго значение „критического“ индекса $\beta$, характеризующего модель фазового перехода, произошедшего при $0.3 \mathrm{GPa}$, эта зависимость была построена в двойном логарифмическом масштабе и оказалась линейной (рис. 5), из наклона которой прямо следует значение критического индекса $\beta=0.4 \pm 0.02$.

Полученное значение, в принципе, не противоречит модели фазового перехода, происходящего вблизи трикритической точки, полученной ранее из температурного поведения аналогичной мягкой моды, наблюдаемой ранее [3-5].

Таким образом, в результате изучения спектров КР кристаллов $\mathrm{Hg}_{2} \mathrm{Br}_{2}$ при высоких гидростатических

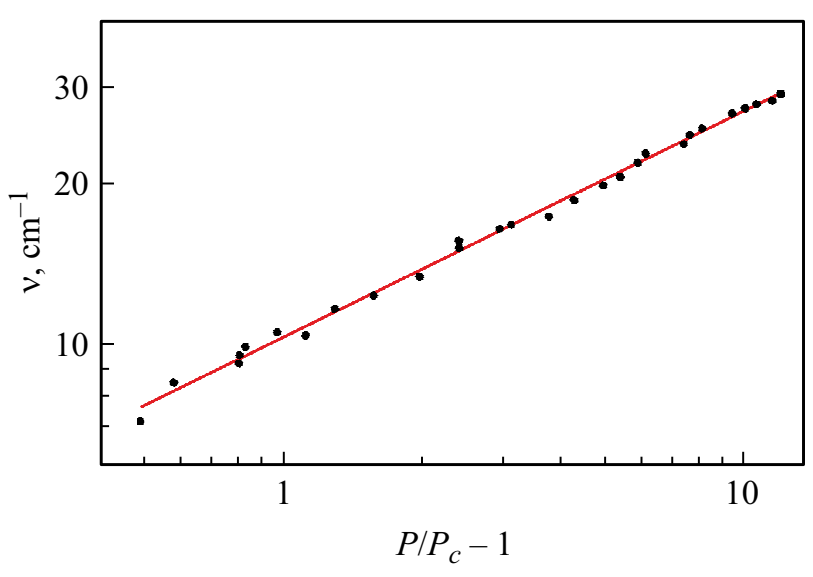

Рис. 5. Барическое поведение мягкой моды в кристаллах $\mathrm{Hg}_{2} \mathrm{Br}_{2}$ в двойном логарифмическом масштабе и от приведенного давления $v_{\mathrm{sm}}(p)$, где $p=P / P_{c}-1$. 
давлениях обнаружено „возгорание“ в спектрах КРфононов, в том числе мягких, выполнена интерпретация и обсуждены полученные результаты, подтверждена модель фазового перехода $D_{4 h}^{17} \rightarrow D_{2 h}^{17}$, предложенная для случая фазового перехода, индуцированного понижением температуры $\mathrm{Hg}_{2} \mathrm{Br}_{2}[3-5]$.

Авторы выражают глубокую признательность Ю.Э. Китаеву за плодотворное обсуждение результатов работы.

\section{Список литературы}

[1] H. Mark, J. Steinbach. Z. Kristallogr. 64, 78 (1926).

[2] Proc. 2nd Int. Symposium on Univalent Mercury Halides. ŠFR Trutnov (1989).

[3] Ч. Барта, А.А. Каплянский, В.В. Кулаков, Б.З. Малкин, Ю.Ф. Марков. ЖЭТФ 70, 429 (1976).

[4] А.А. Каплянский, Ю.Ф. Марков, Ч. Барта. Изв. АН СССР. Сер. физ. 43, 1641 (1979).

[5] Б.С. Задохин, Ю.Ф. Марков, А.С. Юрков. ЖЭТФ 104, 2799 (1993).

[6] Ч. Барта, А.А. Каплянский, Ю.Ф. Марков, В.Ю. Мировицкий. ФТТ 27, 2500 (1985).

[7] Ю.Ф. Марков, А.Ш. Тураев. Письма ЖЭТФ 63, 227 (1996).

[8] Ю.Ф. Марков, В.Ю. Мировицкий, Е.М. Рогинский. ПЖТФ 40, 22, 12 (2014).

[9] Ю.Ф. Марков, В.Ю. Мировицкий, Е.М. Рогинский. ФТТ 57, 3, 469 (2015).

[10] Е.М. Рогинский, А.С. Крылов, Ю.Ф. Марков, М.Б. Смирнов. Изв. РАН. Сер. физ. 80, 9, 1132 (2016).

[11] F. Datchi, A. Dewaele, P. Loubeyre, R. Letoullec, Y.Le Godec, B. Canny. High Pressure Res. 27, 447 (2007).

[12] M.I. Aroyo, A. Kirov, C. Capillas, J.M. Perez-Mato, H. Wondratschek. Acta Crystallogr. A62, 115 (2006); www.cryst.ehu.es

[13] Е.М. Рогинский, Ю.Ф. Марков, М.Б. Смирнов. ФТТ 57, 3, 456 (2015).

Редактор Ю.Э. Китаев 